\title{
V-Series Nerve Agent CVX
}

National Cancer Institute

\section{Source}

National Cancer Institute. V-Series Nerve Agent CVX. NCI Thesaurus. Code C161533.

An organophosphate nerve agent developed by China that is the structural isomer of the nerve agent $V X$, an acetylcholinesterase inhibitor and neurotoxin. 